\section{LAS MICOTOXINAS: EL ENEMIGO SILENCIOSO}

\author{
Antonio Javier Ramos Girona \\ Universitat de Lleida \\ ORCID iD: https://orcid.org/0000-0002-2830-8299 \\ antonio.ramos@udl.cat \\ Sonia Marín Sillué \\ Universitat de Lleida \\ ORCID iD: https://orcid.org/0000-0002-0714-6155 \\ sonia.marin@udl.cat \\ Francisco Molino Gahete \\ Universitat de Lleida \\ ORCID iD: https://orcid.org/0000-0002-1286-3482 \\ francisco.molino@udl.cat \\ Pilar Vila Donat \\ Universitat de Lleida \\ ORCID iD: https://orcid.org/0000-0002-1496-3508 \\ pilar.viladonat@tecal.udl.cat \\ Vicente Sanchis Almenar \\ Universitat de Lleida \\ ORCID iD: https://orcid.org/0000-0001-9889-8098 \\ vicente.sanchis@udl.cat
}

Cómo citar este artículo/Citation: Ramos Girona, A. J., Marín Sillué, S., Molino Gahete, F., Vila Donat, P. y Sanchis Almenar, V. (2020). Las micotoxinas: el enemigo silencioso. Arbor, 196 (795): a540. https://doi.org/10.3989/arbor.2020.795n1004

Recibido: 26 febrero 2019. Aceptado: 29 octubre 2019.

RESUMEN: Las micotoxinas son metabolitos fúngicos secundarios que pueden ejercer un efecto tóxico tanto en el hombre como en los animales debido, principalmente, a su exposición a través de los alimentos. La presencia de estos compuestos ha sido demostrada en una amplia variedad de materias primas, alimentos y piensos, en los que lo habitual es encontrar de forma frecuente una contaminación múltiple por diferentes micotoxinas, en pequeñas cantidades, lo que puede generar efectos tóxicos subcrónicos, así como bioacumulación. Este artículo revisa los principales elementos que configuran la problemática de las micotoxinas para el hombre y los animales, y aborda los retos de futuro que se plantean en el estudio de las micotoxinas, entre los que destacan el efecto que el cambio climático puede tener sobre el patrón de contaminación por micotoxinas en los alimentos, el descubrimiento creciente de nuevas micotoxinas en formas modificadas, la evaluación de la coexistencia de estas toxinas y otros contaminantes, y las formas para detectar e intentar eliminar estos compuestos tóxicos de los alimentos.

PALABRAS CLAVE: micotoxinas; aflatoxinas; mohos filamentosos; cambio climático; exposición; detoxificación,

\section{MYCOTOXINS: THE SILENT ENEMY}

Copyright: (C) 2020 CSIC. Este es un artículo de acceso abierto distribuido bajo los términos de la licencia de uso y distribución Creative Commons Reconocimiento 4.0 Internacional (CC BY 4.0).

ABSTRACT: Mycotoxins are secondary fungal metabolites that can have toxic effects on both human and animals, mainly due to their exposure through food. Presence of these compounds has been demonstrated in a wide variety of raw materials, foods and feeds, in which it is common to find co-occurrence of different mycotoxins, in small amounts, which can generate subchronic toxic effects, as well as bioaccumulation. This article reviews the main elements that shape the problem of mycotoxins for human and animals, and explains the future challenges that will arise in the study of mycotoxins, among which are the effects that climate change can have on the pattern of contamination by mycotoxins in food, the growing discovery of new modified mycotoxins, the evaluation of the co-occurrence of these toxins and other chemical contaminants, and the ways to detect and eliminate these toxic compounds from food.

KEYWORDS: mycotoxins; aflatoxins; moulds; climatic change; exposure; detoxification. 
La industria alimentaria emplea frecuentemente mohos filamentosos en la fabricación de diferentes tipos de alimentos, como es el caso del queso roquefort o el de los embutidos crudos curados, pero, por otra parte, este mismo tipo de microorganismos son contaminantes habituales de ciertos tipos de alimentos, en los que, si las condiciones son las adecuadas, pueden llegar a producir toxinas, a las que denominamos micotoxinas. Este artículo explica cuáles son las características principales de estos metabolitos tóxicos y expone cuáles son los retos a los que habrá de enfrentarse en este ámbito la industria alimentaria en un futuro cercano.

\section{PERO ¿QUÉ SON LAS MICOTOXINAS?}

Las micotoxinas son toxinas producidas principalmente por algunas especies de mohos pertenecientes a los géneros Aspergillus, Penicillium, Fusarium, Claviceps y Alternaria que contaminan alimentos como cereales, frutas y frutos secos, semillas oleaginosas, especias y piensos.

Estos compuestos son metabolitos fúngicos secundarios que están clasificados como peligros químicos de origen biológico, y se ha demostrado que pueden causar daños a la salud de las personas y de los animales. El término empleado para definir la intoxicación por estas toxinas es el de micotoxicosis, y sus efectos nocivos pueden ser originados tanto si se inhalan como si entran en contacto directo con el organismo o son ingeridos con los alimentos contaminados.

No se trata de un riesgo nuevo. Los primeros datos documentados de estas intoxicaciones datan de finales del primer milenio, y corresponden a la enfermedad conocida como Fuego de San Antonio, también conocido como ergotismo, enfermedad que afectó a un gran número de personas en Europa en la Edad Media. Esta intoxicación es causada por el consumo de centeno contaminado con alcaloides producidos por Claviceps purpurea. En épocas más recientes destaca la muerte de más de 100.000 pavos ocurrida en el Reino Unido en 1960 cuando estos fueron alimentados con un pienso a base de harina de cacahuete procedente de Brasil que contenía metabolitos secundarios producidos por Aspergillus flavus, y que, posteriormente, fueron denominados aflatoxinas por estar producidos por esta especie fúngica. Estas micotoxinas fueron los primeros compuestos identificados como tales. Posteriormente, se han ido identificando otras toxinas producidas por diferentes especies fúngicas, y en la actualidad se conocen más de 300 micotoxinas, entre las que destacamos las aflatoxinas (AFs), la ocratoxina A (OTA), la patulina (PAT), la citrinina (CIT), los tricotecenos (TCTs) del tipo A como la toxina T-2 (T2) y la toxina HT-2 (HT2), los tricotecenos del tipo B como el deoxinivalenol (DON), el 3-acetildeoxinivalenol (3Ac-DON) y el 15-acetildeoxinivalenol (15Ac-DON), las fumonisinas (FBs), la zearalenona (ZEN), y los alcaloides ergóticos del cornezuelo de centeno (Marin, Ramos, CanoSancho y Sanchís, 2013).

La clasificación de las diferentes micotoxinas ha sido realizada de acuerdo a múltiples criterios, entre los que destacamos, entre otros, el género fúngico que la produce (micotoxinas de Aspergillus, de Penicillium, de Fusarium, de Alternaria...), la fase de formación en el alimento (micotoxinas de campo o precosecha, y de almacén o poscosecha), sus estructuras químicas (lactonas, cumarinas, sesquiterpenos...), el principal órgano o sistema al que afectan (hepatotoxinas, neurotoxinas, nefrotoxinas, inmunotoxinas..), los efectos que provocan (teratogénicas, mutagénicas, cancerígenas, alergénicas...) o sus orígenes biosintéticos (policétidos, derivados de aminoácidos...).

\section{¿DÓNDE SE ENCUENTRAN LAS MICTOXINAS?}

Las micotoxinas pueden aparecer a lo largo de toda la cadena alimentaria, desde los cultivos en el campo hasta los alimentos procesados, pasando por piensos y alimentos crudos o sin procesar. Su amplia presencia en alimentos y piensos produce importantes pérdidas económicas. No obstante, los cereales, frutos secos, semillas oleaginosas, frutas deshidratadas, café, cacao, especias, y sus respectivos derivados, son los alimentos en los que con mayor frecuencia se han detectado concentraciones importantes de micotoxinas, lo que los hace no ser aptos para su consumo. La tabla 1 muestra las micotoxinas más importantes desde el punto de vista económico y de seguridad alimentaria, con las principales especies fúngicas productoras y los alimentos afectados. Una prueba de la importancia de esta contaminación es que la Food and Agriculture Organization (FAO) estimó que el $25 \%$ de las cosechas mundiales pueden verse afectadas por las micotoxinas, con pérdidas anuales de alrededor de un billón de toneladas de alimentos (véase Manual on the application of the HACCP system in mycotoxin prevention and control). De hecho, hoy en día es el peligro con mayor número de notificaciones de rechazo en frontera y uno de los peligros con mayor número total de notificaciones declaradas por la Autoridad Europea de Seguridad Alimentaria (EFSA) (véase The Rapid Alert System for Food and Feed). 
La contaminación de los alimentos procesados por estas micotoxinas suele proceder mayoritariamente de la contaminación natural de sus materias primas o ingredientes, debido a que no suelen destruirse durante las diferentes etapas de elaboración. Sin embargo, la contaminación de los alimentos de origen animal procede del consumo de piensos contaminados por parte de los animales de granja. En este último caso se trata de una contaminación indirecta. Esto explicaría que alimentos como pan, pasta, aperitivos, cereales de desayuno, café, vino, cerveza, zumos de manzana, compotas, leche, carne o huevos puedan ser una fuente de exposición a este peligro.

Hay que constatar que la coexistencia de varias micotoxinas en un mismo alimento es un hallazgo habitual. Este hecho se fundamenta en que una misma especie fúngica puede producir varias micotoxinas, y que un mismo alimento puede estar contaminado por más de una especie fúngica. Todo esto conlleva que, a pesar de que existan pocos trabajos que estudian esta copresencia en un mismo alimento, cuando se investiga es frecuente encontrar esta multicontaminación. Smith, Madec, Coton y Hymery (2016) realizaron una revisión bibliográfica sobre trabajos publicados en revistas científicas en los que se describe esta copresencia y constataron que, en cereales y productos derivados, de las 127 combinaciones de micotoxinas descritas en la literatura científica, las más observadas fueron AFs+FBs, DON+ZEN, AFs+OTA y FBs+ZEN.

\section{¿SON REALMENTE PELIGROSAS PARA EL HOMBRE?}

No todas las micotoxinas son igualmente peligrosas. La Agencia Internacional de Investigación sobre el Cáncer (IARC) ha clasificado las micotoxinas en función de su relación con la producción de cáncer. Por ejemplo, las cuatro aflatoxinas (AFs) principales (AFB1, AFB2, AFG1 y AFG2) pertenecen al grupo 1, esto es, micotoxinas de las que se han encontrado suficientes evidencias para asegurar su carcinogeneidad en humanos, especialmente debido a su relación con el carcinoma hepatocelular. De hecho, la AFB1 es el agente cancerígeno de origen natural más potente conocido hasta la fecha. Por su parte, la aflatoxina $\mathrm{M}_{1}$ (AFM1), las FBs y la OTA se encuentran en el grupo $2 \mathrm{~B}$, lo que supone que existe una evidencia limitada de una asociación de estos compuestos con el cáncer en seres humanos (calificándose por ello como posiblemente carcinogénicos para humanos), mientras que la ZEN, el DON, las toxinas T2/HT2, la PAT y la CIT se clasifican en el grupo 3, con limitadas o insuficientes evidencias en animales (siendo por ello no clasificables como carcinogénicas para humanos) (Marin et al., 2013).
Los órganos afectados por los efectos tóxicos de las micotoxinas son muy variados, y es habitual que el efecto sea multiorgánico. Por otra parte, desde hace tiempo se sabe que estos compuestos generan deficiencias en el funcionamiento del sistema inmune (Pestka y Bondy, 1990), provocando daños insidiosos más difíciles de evaluar, lo que hace que los individuos afectados se vean más expuestos a infecciones secundarias.

Los síntomas que una intoxicación por micotoxinas pueden comportar son extremadamente variados, lo que suele conllevar una dificultad añadida al diagnóstico de una micotoxicosis. Así, dependen de factores como la especie afectada, la edad, el sexo, su estatus nutricional, etc. Generalizando, se podría decir que en el hombre o en los animales las AFs causan carcinogénesis, daño hepático agudo, cirrosis y teratogénesis, la OTA nefropatía, vómitos, teratogénesis, mutagénesis y embriotoxicidad, las FBs neurotoxicidad, nefrotoxicidad, edema pulmonar y cerebral, hepatotoxicidad y lesión cardiaca, la ZEN síndrome estrogénico y problemas reproductivos, la PAT trastornos gastrointestinales y neurológicos, temblores corporales y mutagenicidad, y el gran grupo de los TCTs toda una serie de síntomas complejos que incluyen rechazo del alimento, vómitos, diarrea, pérdida de atención, hemorragias, edemas, taquicardia, desórdenes sanguíneos, alteración del sistema nervioso, lesiones necróticas cutáneas o bucales, etc., aunque la aparición de cada uno de los síntomas anteriormente enumerados depende en gran medida de la especie animal afectada (Marin et al., 2013).

Hay que destacar que los casos de toxicidad aguda suelen estar más presentes en animales que en seres humanos, estando en este último caso ligados principalmente a condiciones socioeconómicas débiles, por deficiencias en el abastecimiento de alimentos o en el control de la seguridad alimentaria. Se han descrito casos de brotes agudos de aflatoxicosis en humanos, como el que aconteció en Kenia en 2004 que originó 317 afectados, con un total de 125 fallecimientos (39,4\% de mortalidad) (Lewis et al., 2005). No obstante, tanto en animales como en seres humanos lo normal es la toxicidad crónica múltiple, es decir, la ingesta prolongada en el tiempo de dosis pequeñas de muchas micotoxinas de forma simultánea, aportadas por los diferentes componentes de la dieta. Los efectos sinérgicos que las combinaciones de dichas toxinas pueden tener sobre la toxicidad final de estos compuestos es algo que aún está lejos de estar completamente comprendido y que puede condicionar en el futuro una revisión del marco legislativo. 


\section{¿QUÉ EFECTO EJERCEN EN LOS ANIMALES?}

En el caso de los animales, e igual que ocurre con otros compuestos fisiológicamente activos, las micotoxinas son capaces de inducir efectos agudos y crónicos. Generalizando, en las micotoxicosis agudas en animales se dan episodios de hepatitis, hemorragias, nefritis, necrosis del epitelio oral y entérico y muerte. Las micotoxicosis crónicas acarrean una disminución de la productividad en forma de una tasa de crecimiento más lenta, una producción láctea reducida, una tasa de supervivencia inferior y una calidad inferior del animal en el mercado. El consumo de niveles bajos de micotoxinas a través de los piensos, debido a la supresión en la respuesta inmune humoral, predispone al animal a padecer infecciones bacterianas, parasitarias y víricas (Koynarski et al., 2007; Stoev, Goundasheva, Mirtcheva y Mantle, 2000). En términos generales, las micotoxinas, o sus metabolitos tóxicos, pueden ocasionar en los animales inmunosupresión, carcinogénesis, teratogénesis, y cuadros clínicos de hepatotoxicidad, nefrotoxicidad, mielotoxicidad, neurotoxicidad, toxicidad pulmonar y endocrina, siendo los mecanismos más importantes para la aparición de tales manifestaciones el estrés oxidativo, la genotoxicidad y ciertas enfermedades crónicas (Hope, 2013; Kolpin et al., 2014).

En los animales de granja se han descrito varios síndromes específicos relacionados con la exposición a algunos tricotecenos, ZEN o FBs. Estos síndromes cursan con inapetencia, vómitos, anorexia, lesiones orales y gastrointestinales en el primer caso, con problemas reproductivos en el segundo y con leucoencefalomalacia equina y edema pulmonar porcino en el último. Peces, conejos, pollos y cerdos son más sensibles a las micotoxicosis que los rumiantes (siendo el ganado bovino más sensible que el ovino o caprino), debido probablemente a la capacidad degradativa de las micotoxinas por parte de la microbiota ruminal.

La inmensa mayoría de los estudios en los que se ha visto cuáles son los efectos de las micotoxinas en la salud y en la productividad de los animales se centran en el efecto aislado de una única micotoxina, pero es interesante plantear que, si en alimentos para animales se ha dado una alta coincidencia de varias micotoxinas y sus metabolitos, no es descabellado suponer que los cuadros de micotoxicosis aparecerán por efecto de varias micotoxinas a la vez.

Cuando las micotoxinas afectan al crecimiento y a los niveles de producción, la rentabilidad también se ve alterada. Sobre la base de un meta-análisis de la literatura científica realizado por Alltech Mycotoxin Management, se ha estimado el vínculo entre el riesgo de micotoxinas y la rentabilidad animal. Poniendo como ejemplo el nivel promedio de riesgo de micotoxinas determinado en el informe de Alltech North America Harvest Analysis en 2015 y las ecuaciones de predicción derivadas de un resumen de varias publicaciones de investigación, los cerdos pueden tener una pérdida potencial de ganancia diaria de 6,8 gramos por día. Esta pérdida de ganancia equivale a una disminución total de 3,4 kilogramos por cerdo durante un período de 140 días. La rentabilidad de las aves de corral también puede verse afectada por las micotoxinas. El aumento de peso se puede reducir en tres gramos por ave, mientras que en el caso de las gallinas de puesta se ha observado una reducción en cuatro huevos por ciclo productivo, y además el peso de los mismos también se ve reducido en término medio en 0,2 gramos por huevo. Para las vacas lecheras, se ha estimado que tienen una pérdida en la producción de leche de 0,6 litros por vaca y día (véase "The costs of mycotoxins in animal production").

\section{¿SON UN PROBLEMA EN ESPAÑA?}

Durante los últimos veinte años se ha publicado un gran número de trabajos acerca de la presencia de micotoxinas en alimentos procedentes del mercado español. Las muestras analizadas van desde alimentos de gran consumo, como cereales y derivados, leche y derivados y vino a alimentos de menor consumo, como especias, frutas secas, chufas y productos a base de manzana. Las micotoxinas investigadas han sido también muy numerosas, analizando no solo las controladas por la legislación europea (AFs, PAT, OTA, FBs, DON, ZEN) sino también otras, como T2, HT2, nivalenol (NIV), diacetoxiscirpenol (DAS), alternariol $(\mathrm{AOH})$, alternariol monometil éter (AME) y las denominadas micotoxinas emergentes (eniatinas y beauvericina).

En líneas generales, el porcentaje de muestras contaminadas por micotoxinas es elevado pero la contaminación encontrada no supera los límites establecidos por la legislación europea (si la hay). La mayoría de estos estudios realizan un cálculo de la ingesta de estas micotoxinas por parte de la población de nuestro país, no superando la Ingesta Diaria Tolerable (IDT o TDI) propuesta por la UE. Por el contrario, la contaminación por micotoxinas, principalmente, DON, ZEN y FBs en materias primas destinadas para alimentación animal, así como en el propio pienso, es impor- 
tante (Castella, Bragulat y Cabañes, 1999; Griessler, Rodrigues, Handl y Hofstetter, 2010), pero en la UE no existen niveles máximos permitidos de micotoxinas para este material, excepto en el caso de la AFB1.

En relación con la exposición humana a las micotoxinas, es de destacar el estudio de dieta total realizado por nuestro grupo para la Agencia Catalana de Seguridad Alimentaria (ACSA) durante el periodo 20082009 (Cano-Sancho, Marín, Ramos y Sanchís, 2014). El diseño del estudio se basó en la combinación de los datos de consumo de alimentos con los datos de contaminación de los mismos por las micotoxinas objeto del estudio (AFs, OTA, PAT, FBs, ZEN, DON, T2 y HT2). Los primeros datos se obtuvieron de un estudio diseñado y desarrollado específicamente para evaluar la ingesta de los principales alimentos relacionados con las micotoxinas por parte de 1.387 individuos residentes en Cataluña pertenecientes a todos los grupos de edad. Los datos de contaminación se obtuvieron de un amplio estudio en el cual se determinaron los niveles de las micotoxinas en los principales alimentos que podían estar contaminados de acuerdo a la bibliografía. El número total de muestras fue de 3.447 , adquiridas en establecimientos comerciales de las doce ciudades con mayor población de la comunidad, lo que representa el $72 \%$ de la población total de la misma. En estas poblaciones se seleccionaron seis establecimientos y en cada uno de ellos se adquirieron tres productos de cada una de las categorías seleccionadas. Paralelamente, se realizó un estudio de biomarcadores de OTA, DON y FBs en muestras de sangre y orina de voluntarios sanos.

Las conclusiones más importantes de este estudio fueron: a) la presencia de micotoxinas en los alimentos procedentes del mercado es, en general, baja. Las micotoxinas encontradas con mayor frecuencia son el DON, las FBs y la OTA; b) los niveles de concentración encontrados en los alimentos se están por debajo del límite establecido por la Comisión Europea; c) los grupos de población más expuestos son los bebés, los niños y el colectivo de inmigrantes, y d) en todos los casos, los niveles de exposición a estas micotoxinas se encuentran dentro de los límites de seguridad.

Un estudio piloto sobre la evaluación de exposición realizado por Rodríguez-Carrasco, Moltó, Mañes y Berrada (2014) en 54 muestras de orina de niños y adultos residentes en Valencia mostró que la exposición al DON era importante, confirmando el resultado obtenido en el estudio de Cano-Sancho et al. (2014).

Como hemos explicado, la contaminación por micotoxinas en los alimentos, aunque en niveles bajos, es elevada, contaminación que ha de sumarse a la presencia de otros compuestos químicos que pueden estar igualmente presentes, como pesticidas, metales pesados, etc. En estos momentos hay muy poco descrito sobre las posibles interacciones sinérgicas que pueda haber entre todos estos tipos de contaminantes, estudios que se enmarcarían dentro de un concepto más amplio denominado exposoma (Marín, Cano-Sancho, Sanchís y Ramos, 2018).

\section{¿CUÁLES SON LOS RETOS DE FUTURO EN ESTE CAMPO?}

Aunque muchos pueden ser los temas que van a mover la investigación en un futuro cercano en el campo de las micotoxinas, hay tres aspectos que consideramos clave para el desarrollo de este campo: la relación entre las micotoxinas y el cambio climático, la determinación de la contaminación de los alimentos por micotoxinas modificadas, y el estudio de las interacciones entre las micotoxinas y otros contaminantes concurrentes en un mismo alimento (exposoma). En adelante trataremos estos tres aspectos con más detalle.

\section{Cambio climático}

El clima afecta profundamente al crecimiento, distribución, y producción de micotoxinas por parte de los mohos. Por ello, el cambio climático puede potencialmente hacer variar el riesgo que los mohos micotoxigénicos suponen para la seguridad alimentaria (Medina, González-Jartin y Sainz, 2017). La aparición de nuevas combinaciones micotoxina-cultivo es preocupante y señala la posibilidad de aparición de nuevos genotipos fúngicos, más agresivos y con diferente potencial micotoxigénico.

Hasta 2004 la perspectiva europea sobre la contaminación por AFs se limitaba solo a los alimentos importados. Varias encuestas realizadas antes de esa fecha para detectar AFs en muestras de alimentos en Europa solo mostraban un pequeño porcentaje de alimentos contaminados con concentraciones de AFB1 por encima del límite reglamentario. Sin embargo, más tarde, un amplio estudio realizado por la Autoridad Europea de Seguridad Alimentaria (EFSA) estableció el problema emergente de la posible contaminación por AFs de maíz, almendras y pistachos cultivados en áreas del sur de Europa, debido al clima subtropical de los últimos años (véase Opinion of the Scientific Panel on Contaminants in the Food Chain on a request from the Commission related to the potential increase of consumer health risk by $a$ possible increase of the existing maximum levels for aflatoxins in almonds, hazelnuts and pistachios and 
Tabla 1. Principales micotoxinas desde el punto de vista económico y de seguridad alimentaria, con sus principales especies fúngicas productoras y alimentos afectados

\begin{tabular}{|c|c|c|}
\hline Micotoxina & Especies productoras & Alimentos \\
\hline $\begin{array}{l}\text { Aflatoxinas } B_{1}, B_{2} \\
G_{1} \text { y } G_{2}\end{array}$ & $\begin{array}{l}\text { Aspergillus sección Flavi (A. flavus, } \\
\text { A. parasiticus, A.nomius). }\end{array}$ & $\begin{array}{l}\text { Cereales y derivados (principalmente maíz), frutos } \\
\text { secos y derivados, frutas deshidratadas, especias }\end{array}$ \\
\hline Ocratoxina A & $\begin{array}{l}\text { Aspergillus sección Circumdati } \\
\text { (A. ochraceus, A. steynii A. westerdijkiae). } \\
\text { Aspergillus sección Nigri (A. carbonarius). } \\
\text { Penicillium verrucosum, P. nordicum. }\end{array}$ & $\begin{array}{l}\text { Cereales y derivados (principalmente trigo y arroz), } \\
\text { café, cacao, vino, frutas deshidratadas, especies) }\end{array}$ \\
\hline Patulina & $\begin{array}{l}\text { Penicillium expansum. Byssochlamys } \\
\text { nívea. Aspergillus clavatus. }\end{array}$ & Manzana, pera y derivados \\
\hline Citrinina & $\begin{array}{l}\text { Monascus purpureus, } M . \text { ruber. } \\
\text { Penicillium verrucosum, P. citrinum. }\end{array}$ & Cereales y derivados (principalmente arroz), frutas \\
\hline Alcaloides ergóticos & $\begin{array}{l}\text { Claviceps purpurea, C. fusiformis, } \\
\text { C. africana. }\end{array}$ & Cereales y derivados (principalmente centeno) \\
\hline Fumonisinas $\mathrm{B}_{1}, \mathrm{~B}_{2}$ y $\mathrm{B}_{3}$ & $\begin{array}{l}\text { Fusarium sección Liseola (F. verticillioides, } \\
\text { F. proliferatum, F. nygamai). }\end{array}$ & Cereales y derivados (principalmente maíz) \\
\hline Zearalenona & $\begin{array}{l}\text { Fusarium graminearum, F. culmorum, } \\
\text { F. incarnatum, F. sporotrichioides. }\end{array}$ & Todos los cereales y derivados \\
\hline Deoxinivalenol & $\begin{array}{l}\text { Fusarium graminearum, F. culmorum, } \\
\text { F. sporotrichioides. }\end{array}$ & Todos los cereales y derivados \\
\hline
\end{tabular}

Fuente: adaptado de Marin et al., 2013.

derived products). De hecho, podría esperarse un cambio en las áreas de presencia tradicional de las AFs debido al aumento de las temperaturas promedio. En este sentido, las zonas mediterráneas se han identificado como un punto donde se prevén cambios extremos en la temperatura, los niveles de $\mathrm{CO}_{2}$ y los patrones de lluvias.

Con respecto a las AFs, aparecen más frecuentemente en épocas calurosas y de sequía, lo que puede estresar a la planta huésped y, por lo tanto, facilitar la infección por A. flavus. Así, en los últimos 15 años, varias temporadas cálidas y secas han dado lugar a graves infecciones por $A$. flavus en el maíz en varios países de Europa (como Italia, Rumanía, Serbia y España). En el escenario de cambio climático de $+2{ }^{\circ} \mathrm{C}$, se predice un claro aumento del riesgo de AFs en áreas tales como el centro y el sur de España, el sur de Italia, Grecia, el norte y el sureste de Portugal, Bulgaria, Albania, Chipre y la Turquía europea, en comparación con lo que ocurriría si se mantuviera la temperatura actual. Además del alto riesgo de AFs en estos países del sur de Europa, se predice un riesgo bajo y medio de AFs en la cosecha en los cuatro principales países productores de maíz (Rumanía, Francia, Hungría y el noreste de Italia) (Battilani et al., 2016).
Con respecto a las toxinas de Fusarium, Europa, que se caracteriza por una amplia gama de condiciones climáticas desde el sur (países mediterráneos) hasta las regiones central y norte, muestra diferencias importantes en la composición de especies asociadas con la fusariosis en cereales (Fusarium Head Blight, FHB). Si bien $F$. graminearum es el principal productor de DON en Europa central y meridional, F. culmorum es dominante en las raíces de los cereales, la base del tallo y las espigas en todos los países escandinavos, incluida Finlandia (Logrieco y Moretti, 2008). Sin embargo, en la última década se ha observado una disminución en la presencia de F. culmorum y un aumento en F. graminearum en algunas áreas del centro y norte de Europa (Nielsen et al., 2011). En general, en las regiones más al sur de Italia y de España, la incidencia de FHB es baja o está ausente; sin embargo, en las regiones más septentrionales de Italia, España y Portugal, el sur de Francia y toda la península balcánica, se observa que F. graminearum ocurre con frecuencia en los cereales en la madurez, junto con el DON (Logrieco y Moretti, 2008). Por otro lado, Madgwick et al. (2011) estudiaron el impacto del cambio climático en la fecha de antesis del trigo y concluyeron que las epidemias de FHB serán más graves, especialmente en el sur de Inglate- 
rra, debido al aumento de F. graminearum y el DON asociado. Otro estudio realizado en los Países Bajos evaluó los efectos del cambio climático proyectados para 2040. El estudio reveló que la mejora de la floración del trigo y la maduración total en una a dos semanas en la mayor parte del noroeste de Europa podría resultar en un aumento de la contaminación por DON, en hasta un factor de tres, con una estimación de una concentración media de DON en el escenario futuro que excede el límite máximo permitido en la UE (van der Fels-Klerx, van Asselt, Madsen y Olesen, 2013).

\section{Micotoxinas modificadas}

Se denominan micotoxinas modificadas a los derivados de las micotoxinas principales cuya estructura ha cambiado debido a su unión a otras matrices, o a la modificación de su estructura básica ocasionada por modificaciones químicas o biológicas, de forma que suelen ser indetectables por las técnicas analíticas rutinarias. Entre ellas cabe distinguir las denominadas micotoxinas biológica o químicamente modificadas (entre las que se encuentran los conjugados producidos por las plantas, anteriormente denominados masked mycotoxins), que son conjugados solubles, extraíbles y fácilmente detectables cuando se conocen y se tienen patrones, y las micotoxinas asociadas a matriz (también llamadas bound mycotoxins), que son conjugados insolubles, no extraíbles, y más difíciles de analizar, ya que previamente hay que liberarlos de la matriz por métodos químicos o enzimáticos (Rychlik et al., 2014).

Hasta la fecha estas micotoxinas no se han venido analizando en las materias primas o alimentos porque se desconocía su existencia o porque no eran detectadas por los métodos de análisis convencionales, debido principalmente a que el cambio de su estructura molecular altera sus características cromatográficas, modifica el epitopo reconocido por los anticuerpos para su detección por técnicas inmunológicas, o incluso porque la eficacia del disolvente de extracción se ve reducida al haber sufrido la molécula un cambio en su polaridad debido a la modificación, todo lo cual puede conducir a una subestimación del contenido total real de micotoxinas en un alimento.

El hecho de que las plantas produzcan la metabolización de las micotoxinas a sus formas modificadas responde a un mecanismo de detoxificación por el que las plantas convierten micotoxinas relativamente apolares en derivados más polares, que son entonces más fácilmente almacenados en vacuolas, o son conjugados a biopolímeros, como los componentes de la pared celular.
Por otra parte, el procesado de los alimentos puede causar la generación de nuevas micotoxinas modificadas, debido, por ejemplo, a los procesos que implican la aplicación de calor, como ocurre en el caso de la FB1 y la aparición de la N-carboximetil FB1 cuando la FB1 se calienta en presencia de azúcares reductores (Howard, Churchwell, Couch, Marques. y Doerge, 1998). Además, se ha descrito la liberación enzimática de las micotoxinas unidas a la matriz alimentaria, por ejemplo debido a la acción de enzimas usualmente utilizados en la industria alimentaria, como las amilasas empleadas en la industria de la panificación, provocando un incremento en el nivel total de micotoxinas existentes en el producto final con respecto a la contaminación encontrada en la materia prima (Vidal, Morales, Sanchís, Ramos y Marín, 2014), lo que suele ser un resultado bastante inesperado.

En cuanto a las consideraciones toxicológicas de este tipo de micotoxinas, todavía hay mucho por determinar, pero no se puede descartar que en algunos casos puedan ser incluso más tóxicas que las moléculas parentales, sobre todo si presentan una mayor bioaccesibilidad y biodisponibilidad (Freire y Sant'Ana, 2018).

En algunos casos se ha comprobado que la molécula modificada puede regenerar la toxina original en el sistema digestivo por acción de enzimas metabólicos (en el propio sistema digestivo o en otros órganos) o de los microorganismos de la microbiota intestinal (Gratz et al., 2017). Esto puede conllevar la aparición de sintomatologías más graves que las que cabría esperar a la vista de la contaminación de los alimentos que se ingieren, debido a que realmente en el tracto gastrointestinal hay presentes más toxinas de las esperadas. Por ello, hay que considerar la modificación de la legislación para incluir la suma del total de micotoxinas (parental+modificadas) a la hora de establecer los límites legales y realizar la evaluación del riesgo por micotoxinas.

\section{Coexposición a peligros químicos}

Los métodos de detección multimicotoxina han permitido evaluar los niveles de coexposición a diferentes micotoxinas de un mismo individuo a través de un simple análisis de orina. De este modo se ha confirmado que la coexistencia de dos toxinas en una muestra de orina suele ser lo más común, mucho más que la contaminación por una única micotoxina. Sin embargo, los resultados dependen en gran medida de las toxinas analizadas. Así, si solo se analizan las micotoxinas parentales, generalmente se detectan una o dos toxinas en cada muestra de orina, mientras que 
si se analizan las micotoxinas parentales y las modificadas, generalmente se encuentran de dos a cuatro toxinas en una muestra.

Por otra parte, un menor límite de detección de los métodos lleva a detectar una mayor coexistencia. Por ejemplo, Gerding et al. (2015) informaron de que entre el $16-54 \%$ de las muestras de orina contenían dos toxinas detectables, entre el $6-20 \%$ de las muestras contenían tres toxinas y entre el 1-2\% contenían cuatro toxinas. Dihidrocitrinona (DH-CIT) y OTA son dos toxinas que usualmente coexisten, al igual que DON y deoxinivalenol glucurónido (DON-GlcA), así como también tres de ellas. También se ha demostrado coexistencia de las toxinas de Fusarium y OTA (Wallin et al., 2015), por ejemplo, las combinaciones DON-ZENOTA y DON-ZEN-FB1-OTA, se presentaron en el 38 y $52 \%$ de las muestras de orina analizadas (Solfrizzo, Gambacorta y Visconti, 2014).

Una línea de investigación futura importante sería la posible exposición concurrente de micotoxinas con otras sustancias químicas ambientales que pueden mostrar alguna actividad interactiva y ejercer alguna función biológica que converja en las mismas vías moleculares. Por lo que sabemos, no hay estudios que monitoricen la presencia simultánea de un panel de sustancias químicas ambientales, incluido algún tipo de micotoxina. Sin embargo, las estimaciones basadas en modelos de exposición dietética sugieren que es probable que haya múltiples patrones de exposición simultánea dentro de la población general. La investigación sobre la identificación de mezclas del Segundo Estudio de Dieta Total en Francia reveló la exposición prolongada a las micotoxinas en mezclas complejas con otros químicos ambientales en la mayoría de los grupos de dietas francesas. Por ejemplo, se estimó que existía un primer grupo, que comprendía el $18 \%$ de la población total, con una exposición significativamente mayor a las micotoxinas (toxina HT2, DON, ZEN y NIV), hidrocarburos aromáticos policíclicos (HAP) (pireno y fenantreno) y bisfenol A que el resto de la población. La ZEN, micotoxina estrogénica, también se identificó en otro grupo con muchos HAP, acrilamida, oligoelementos, pesticidas y la suma de ocho éteres de difenilo polibromados, en un grupo que representaba al $21 \%$ de la población con hábitos alimenticios relacionados con la denominada "comida basura" (Traore, Bechaux, Sirot y Crepet, 2016).

Esta contaminación múltiple puede modificar fuertemente los efectos tóxicos de algunos de los contaminantes, lo que resulta en una variedad de efectos interactivos, como lo demuestra la exposición simul- tánea de células Caco-2 y células de riñón HEK-293 a cadmio y DON (Le, Alassane-Kpembi, Oswald y Pinton, 2017). La evaluación toxicológica de combinaciones de micotoxinas para la caracterización de interacciones potenciales es un campo de investigación emergente y muy activo.

\section{¿SE PUEDE HACER ALGO PARA CONTROLAR LAS MICOTOXINAS?}

A pesar del tiempo que se llevan estudiando las micotoxinas y buscando una solución a la contaminación por micotoxinas en las materias primas, alimentos y piensos, todavía no existe ningún proceso de detoxificación que haya demostrado ser lo suficientemente seguro y eficiente. De hecho, en la Unión Europea (UE) está prohibido aplicar tratamientos químicos diseñados para la eliminación de este tipo de compuestos. No obstante, las técnicas aplicadas durante la elaboración de los alimentos pueden tener cierta influencia en la degradación de las micotoxinas. Por otra parte, y exclusivamente en el campo de la alimentación animal, el uso de compuestos adsorbentes o biodegradadores es una estrategia que sí ha sido reconocida como efectiva por la UE, de modo que su empleo está autorizado como aditivos para piensos para el control de la AFB1, tal y como veremos más adelante.

El primer objetivo para establecer un control de las micotoxinas es la prevención de su acumulación en el campo y en la poscosecha. Sin embargo, a pesar de los esfuerzos realizados en investigación en este ámbito, a día de hoy las micotoxinas suelen estar presentes en las materias primas utilizadas para la producción de alimentos, y en particular en los productos a base de cereales. Así pues, existe un interés evidente por cuantificar los efectos que ejercen las diferentes operaciones unitarias empleadas habitualmente durante el procesamiento de los alimentos sobre las micotoxinas. Este conocimiento es clave para los estudios de exposición y evaluación de riesgos, los cuales pueden usarse para el desarrollo de estrategias de prevención con base científica con el objetivo de salvaguardar la salud pública.

A pesar de la importancia de comprender los efectos de las operaciones unitarias del procesamiento de alimentos sobre el destino de las micotoxinas, se sabe que la mayoría de estos compuestos son estables en cierto grado a operaciones tales como el calentamiento, extrusión, cocción, horneado, fritura, asado, cocinado, laminado, enlatado y nixtamalización, proceso este último consistente en la cocción del maíz con agua y cal viva muy empleado en México y otras zonas de Centro y Sudamérica. 
Las operaciones unitarias más efectivas para reducir los niveles de micotoxinas, sin embargo, parecen ser las que involucran la eliminación física de las partes enmohecidas de los granos, como la clasificación o limpieza de los mismos (Bullerman y Bianchini, 2007).

La molienda puede reducir la concentración de micotoxinas de diferentes maneras. Se ha comprobado que la molienda da como resultado una distribución desigual de las micotoxinas en las diferentes fracciones obtenidas, como en el caso del salvado, que tiende a acumular micotoxinas, y que en su mayoría se consume como alimento para animales. Simultáneamente, la concentración de micotoxinas en la otra fracción (harina) disminuirá. Además, en la molienda húmeda, la micotoxina se puede disolver en el agua usada y tiende a acumularse en el germen y la fibra de gluten. Por lo tanto, el almidón obtenido tiende a estar relativamente libre de micotoxinas (Bullerman y Bianchini, 2007).

El efecto de la fermentación como un proceso biológico en la reducción de micotoxinas ha sido ampliamente estudiado en los últimos años. La fermentación de la harina y la masa puede causar un aumento en la concentración de DON. Sin embargo, se han observado disminuciones en ZEN, OTA y AFs. Por lo que respecta a DON, se han publicado resultados contradictorios. Bergamini et al. (2010) observaron un aumento significativo en la concentración de DON después de la etapa de fermentación. En contraste, la concentración de DON se redujo en aproximadamente un $41 \%$ y un $56 \%$ en procesos de panificación estudiados en Francia y Austria, respectivamente. Se ha confirmado la reducción efectiva del DON no solo por el tratamiento térmico sino también por el proceso de fermentación (Samar, Neira, Resnik y Pacin, 2001). Los agentes mejorantes y los microorganismos (masa madre), podrían causar diferentes efectos sobre la concentración de micotoxinas. El uso de enzimas parece llevar al aumento de la concentración de DON durante la fermentación. Además, mediante la incorporación de mezclas de enzimas (xilanasa y $\alpha$-amilasa) como ingredientes de la masa, se ha observado un aumento de DON-3-glucósido (DON-3-GIc) en la masa fermentada (Vidal, Ambrosio, Sanchís, Ramos y Marín, 2016). El aumento de las concentraciones de DON y DON-3Glc durante la panificación puede deberse a la actividad enzimática, ya que la hidrólisis de los carbohidratos $u$ otros componentes podría liberar micotoxinas conjugadas (Vidal et al., 2016). Las diferencias entre los estudios podrían estar relacionadas con las condiciones de fermentación empleadas (los microorga- nismos involucrados, como Lactobacillus plantarum, Lactobacillus acidophilus y Saccharomyces cerevisiae, ingredientes y prácticas. Se necesitan más estudios para aclarar los factores que conducen al aumento o a la reducción de los niveles de micotoxinas durante la fermentación de los alimentos, ya que estos incluyen datos clave para la corrección de los modelos de evaluación de riesgos.

En general, el tratamiento térmico se emplea comúnmente para la descontaminación microbiológica, ejerciendo a la vez una actividad sensorial y nutricional. Teniendo en cuenta la estabilidad térmica y química relativamente alta de las micotoxinas, con la aplicación de calor se logran diferentes resultados en términos de su reducción. Las AFs son muy estables a temperaturas superiores a $100^{\circ} \mathrm{C}$, por lo que se puede lograr poca o casi ninguna descomposición de las mismas mientras se someten a pasteurización, tostado y horneado (Campagnollo et al., 2016). Sin embargo, pueden reducirse (78-88\%) mediante tratamiento térmico a presión (Milani y Maleki, 2014). El DON es una micotoxina estable durante el tratamiento térmico hasta $120-180^{\circ} \mathrm{C}$ y parcialmente estable a $210^{\circ} \mathrm{C}$ (Jard, Liboz, Mathieu, Guyonvarc'h y Lebrihi, 2011). Con respecto a la cocción, algunas investigaciones han demostrado que el DON podría reducirse durante el horneado del pan, y que dicha reducción se ve afectada por los aumentos en la temperatura y el tiempo de horneado. Sin embargo, según otras investigaciones, se ha visto una reducción más baja de DON durante la cocción, que puede estar asociada con el tamaño del pan o con un tratamiento térmico suave.

Además, como resultado del procesamiento de alimentos, las micotoxinas pueden presentar algunas reacciones con los ingredientes incorporados, como los azúcares reductores, o incluso unirse a varios componentes dentro de la matriz alimentaria. Por lo tanto, la transformación a una forma modificada es otra posible razón para la reducción o el aumento aparente de algunas de las micotoxinas investigadas, cuyos efectos toxicológicos no son bien conocidos. Cabe destacar que la desaparición de las micotoxinas en los resultados analíticos no significa necesariamente su ausencia real o, de hecho, una disminución de la toxicidad debida a este tipo de compuestos, ya que en ocasiones los productos de descomposición producidos pueden ser tan peligrosos como las micotoxinas parentales.

Si bien la prevención de la contaminación por micotoxinas en el campo y durante el almacenamiento es el objetivo principal de la producción primaria y de piensos, la ausencia de micotoxinas en la ración de los 
animales de granja todavía no puede asegurarse por completo (Wielogórska, MacDonald y Elliot, 2016).

Además de las buenas prácticas agrícolas para prevenir la contaminación de los piensos con micotoxinas, se han desarrollado otras estrategias con la finalidad de reducir la absorción de micotoxinas en el tracto digestivo mediante el uso de agentes secuestrantes o detoxificantes. Los agentes detoxificantes se definen como «sustancias que pueden suprimir o reducir la absorción, promover la excreción de micotoxinas o modificar su modo de acción» (véase Commission regulation (EC) No. 386/2009 of 12 May 2009). Esta nueva categoría de aditivos, que reducen la absorción de micotoxinas en el tracto gastrointestinal del animal o que modifican su estructura se conocen como agentes adsorbentes o biotransformadores (Jard et al., 2011). El uso de los primeros (distintos tipos de arcillas y silicatos) está más extendido que el de los segundos (enzimas o microorganismos capaces de detoxificar algunas micotoxinas). Estos aditivos se adicionan a la dieta de los animales (principalmente de cerdos, aves y ganado vacuno) para reducir la absorción de las micotoxinas en el tracto digestivo y su distribución a la sangre y los órganos diana. Los adsorbentes se unen a las micotoxinas presentes en los piensos contaminados sin disociarse en el tracto digestivo del animal, lo que limita su biodisponibilidad después de la ingestión, disminuyendo la exposición de los animales a las micotoxinas. De esta manera, el complejo micotoxina-adsorbente pasa a través del animal y es eliminado a través de las heces. Estos agentes se dividen a su vez en dos subgrupos: compuestos inorgánicos y orgánicos (Jard et al., 2011). Los adsorbentes inorgánicos, como los aluminosilicatos (bentonitas, y algunas zeolitas), han demostrado una gran eficacia adsorbiendo AFs (Kong, Shin y Kim, 2014). Sin embargo, no han demostrado ser tan eficaces frente a otro tipo de micotoxinas, como aquellas producidas por el género Fusarium (Harper, Estienne, Meldrum, Harrell y Diaz, 2010; Ramos y Hernández, 1997). De hecho, una bentonita dioctaédrica (Mycofix ${ }^{\circ}$, Biomin, Austria) es, de momento, el único producto autorizado por la Unión Europea como adsorbente de AFB1 (EC 1060/2013). Por otro lado, los adsorbentes orgánicos (paredes celulares de levaduras, fibras micronizadas o bio-sorbentes, como el orujo de uva) están demostrando tener mayor efectividad contra un amplio espectro de micotoxinas (FBs, OTA y ZEN) (Avantaggiato, Greco, Damascelli, Solfrizzo y Visconti, 2014). Solo algunos $\beta$-glucanos y mananos, junto con ciertos polímeros sintéticos (colestiramina) se han identificado como posibles adsorbentes de DON (Faucet-Marquis, Joannis-Cassan, Hadjeba-Medjdoub,
Ballet y Pfohl-Leszkowicz, 2014). La combinación de las propiedades de diferentes adsorbentes (minerales, minerales modificados, orgánicos y sintéticos) podría adaptarse mejor a los casos de piensos multi-contaminados, cada vez más frecuentes, siendo actualmente uno de los retos que hay que abordar (Vila-Donat, Marín, Sanchís y Ramos, 2018).

Otra estrategia para el control de las micotoxinas es su degradación a metabolitos menos tóxicos mediante el uso de agentes biotransformadores. La biotransformación se puede lograr con enzimas que degraden las micotoxinas o mediante el empleo de microorganismos que produzcan dichas enzimas. Varias especies microbianas, incluidas bacterias, levaduras y mohos, han sido reconocidas por su capacidad de transformar las micotoxinas en metabolitos menos tóxicos a través de rutas tales como acetilación, oxigenación, isomerización o glucosilación. La cepa BBSH 797 de Eubacterium, aislada de los fluidos del rumen bovino, ha sido muy estudiada por su capacidad de degradar DON y otros tricotecenos, habiéndose introducido en el mercado con el nombre comercial de Mycofix BBSH (Biomin, Getzersdorf, Austria). Además, algunos de los enzimas responsables de las biotransformaciones descritas en estas especies microbianas se han aislado y aplicado directamente como agentes detoxificantes (Boudergue et al., 2009). No obstante, la falta de información sobre los mecanismos de transformación, la toxicidad de los productos derivados de la biotransformación, el efecto de las reacciones de transformación en los valores nutricionales de los alimentos y la seguridad para los animales hace que su uso no esté todavía demasiado extendido (Wielogórska et al., 2016).

\section{¿LA LEGISLACIÓN NOS PROTEGE?}

Con el descubrimiento de las AFs en la década de los años sesenta del pasado siglo y la constatación de su elevado poder cancerígeno, muy pronto se desarrolló una legislación específica para este tipo de micotoxinas en casi todos los países del mundo desarrollado, legislándose bien los niveles máximos admisibles de la AFB1 en solitario o bien del conjunto de las cuatro AFs principales (AFB1+AFB2+AFG1+AFG2). Igualmente, la presencia de la AFM1 en la leche y derivados lácteos fue sometida pronto a legislación. En la Unión Europea los límites máximos para alimentación humana, varían entre cuatro-quince $\mu \mathrm{g} / \mathrm{kg}$ para las AFs totales y de 0,1-12 $\mu \mathrm{g} / \mathrm{kg}$ para la AFB1, de acuerdo con el tipo de alimento y su destino. En el caso de la leche y sus derivados, los niveles máximos son de 0,025-0,050 $\mu \mathrm{g}$ de $A F M 1 / \mathrm{kg}$ y de $0,1 \mu \mathrm{g}$ de $A F B 1 / \mathrm{kg}$. 
También existe legislación para otras micotoxinas en alimentos para consumo humano, como es el caso de OTA, PAT, DON, ZEN, toxinas T2 y HT2, FBs y CIT (Ramos, Rocha Rosa, Cavaglieri y Guedes, 2011). En cuanto a la alimentación animal, en la UE solo existe legislación para AFB1, con límites máximos entre cinco a veinte $\mu \mathrm{g} / \mathrm{kg}$, según el tipo y destino del pienso. No obstante, la Unión Europea ya ha dictaminado una serie de recomendaciones sobre la presencia de DON, ZEN, OTA, FBs, toxinas T2 y HT2 en productos destinados a alimentación animal (Ramos et al., 2011).

De momento el panorama legislativo en lo referente a las micotoxinas se encuentra estancado. No obstante, es muy posible que, en un medio plazo, la Unión Europea pase a legislar los niveles máximos admisibles del resto de micotoxinas diferentes a la AFB1 en alimentación animal. En un futuro más lejano, sería deseable que la Unión Europea acometiera la legislación referente a los niveles máximos admisibles de las combinaciones de diferentes micotoxinas, habida cuenta de su demostrado efecto sinérgico, así como la presencia en los alimentos y piensos de las micotoxinas modificadas.

\section{CONCLUSIONES}

A pesar de llevar descubiertas más de medio siglo, las micotoxinas siguen siendo una fuente inagotable de sorpresas en el campo de la toxicología humana y animal. La evidencia creciente de que existen muchas más micotoxinas de las que actualmente se conocen, especialmente debido a la existencia de múltiples formas modificadas de difícil detección y de toxicidad todavía poco conocida, así como la conciencia de que su interacción con otros contaminantes habitualmente presentes en los alimentos puede desembocar en efectos sinérgicos negativos, nos aboca a un panorama más complejo que el que se vislumbra en la actualidad. Por otra parte, el efecto que el cambio climático puede tener en un incremento en la contaminación por micotoxinas en Europa es un hecho frente al cual tenemos que prepararnos, especialmente habida cuenta de lo difícil que es eliminarlas de los alimentos una vez se encuentran presentes. Por último, en un futuro próximo, y a la vista de todas estas consideraciones, se debería revisar profundamente la legislación que atañe a la alimentación humana y aumentar el número de micotoxinas que se controlan en alimentación animal, sobre todo teniendo en cuenta el efecto que tienen no solo en la salud y productividad de los animales sino en su posible transferencia a la alimentación humana a través de los alimentos que obtenemos de ellos.

\section{AGRADECIMIENTOS}

Los autores agradecen al Ministerio de Economía y Empresa (MINECO) del Gobierno de España la financiación recibida (proyectos AGL2014-55379 y AGL2017-87755-R).

\section{BIBLIOGRAFÍA}

Avantaggiato, G., Greco, D., Damascelli, A., Solfrizzo, M. y Visconti, A. (2014). Assessment of multi-mycotoxin adsorption efficacy of grape pomace. Journal of Agricultural and Food Chemistry, 62 (2), pp. 497-507. https://doi.org/10.1021/jf404179h

Battilani, P., Toscano, P., Van der Fels-Klerx, H. J., Moretti, A., Camardo Leggieri, M., Brera, C., Portais, A., Goumperis, T. y Robinson, T. (2016). Aflatoxin $B_{1}$ contamination in maize in Europe increases due to climate change. Scientific Reports, 6, 24328. https://doi.org/10.1038/srep24328

Bergamini, E., Catellani, D., Dall'Asta, C., Galaverna, G., Dossena, A., Merchelli, R. y Suman, M. (2010). Fate of Fusarium mycotoxins in the cereal product supply chain: The deoxynivalenol (DON) case within industrial baking process in the bread-making technology. Food Additives and Contaminants, 27 (5), pp. 677-687. https:// doi.org/10.1080/19440041003660117
Boudergue, C., Burel, C., Dragacci, S., Favrot, M. C., Fremy, J. M., Massimi, C. [...] y Avantaggiato, G. (2009). Review of mycotoxin-detoxifying agents used as feed additives: mode of action, efficacy and feed/food safety. EFSA Supporting Publications, 6 (9), 22E. https://doi. org/10.2903/sp.efsa.2009.EN-22

Bullerman, L. B. y Bianchini, A. (2007). Stability of mycotoxins during food processing. International Journal of Food Microbiology, 119 (1-2), pp. 140-146. https://doi.org/10.1016/j.ijfoodmicro.2007.07.035

Campagnollo, F. B., Ganev, K. C., Khaneghah, A. M., Portela, J. B., Cruz, A. G., Granato, D., Corassin, C. H., Oliveira, C. A. F. y Sant'Ana, A. S. (2016). The occurrence and effect of unit operations for dairy products processing on the fate of aflatoxin $\mathrm{M} 1$ : A review. Food Control, 68, pp. 310-329. https://doi. org/10.1016/j.foodcont.2016.04.007
Cano-Sancho, G., Marín, S., Ramos A. J. y Sanchis, V. (2014). Micotoxines. Estudi de dieta total a Catalunya 2008-2009. Barcelona: Agencia de Salut Pública de Catalunya. Disponible en: https://scientiasalut.gencat.cat/bitstream/handle/11351/997/micotoxines_estudi_dieta_catalunya_2014.pdf?sequence $=1$

Castellá, G., Bragulat, M. R. y Cabañes, F. J. (1999). Surveillance of fumonisins in maize-based feeds and cereals from Spain. Journal of Agricultural and Food Chemistry, 47 (11), pp. 4707-4710. https://doi.org/10.1021/jf981236d

Faucet-Marquis, V., Joannis-Cassan, C., Hadjeba-Medjdoub, K., Ballet, N. y Pfohl-Leszkowicz, A. (2014). Development of an in vitro method for the prediction of mycotoxin binding on yeast-based products: case of aflatoxin $B_{1}$, zearalenone and ochratoxin A. Applied Microbiology and Biotechnology, 98 (17), pp. 
7583-7596. https://doi.org/10.1007/ s00253-014-5917-y

Freire, L. y Sant'Ana, A. S. (2018). Modified mycotoxins: An updated review on their formation, detection, occurrence, and toxic effects. Food and Chemical Toxicology, 111, pp. 189-205. https://doi. org/10.1016/j.fct.2017.11.021

Gerding, J., Ali, N., Schwartzbord, J., Cramer, B., Brown, D. L., Degen, G. H. y Humpf, H.-U. (2015). A comparative study of the human urinary mycotoxin excretion patterns in Bangladesh, Germany, and Haiti using a rapid and sensitive LC-MS/MS approach. Mycotoxin Research, 31 (3), pp. 127-136. https://doi.org/10.1007/ s12550-015-0223-9

Gratz, S. W., Dinesh, R., Yoshinari, T., Holtrop, G., Richardson, A. J., Duncan, G., MacDonald, S., Lloyd, A. y Tarbin, J. (2017). Masked trichothecene and zearalenone mycotoxins withstand digestion and absorption in the upper Gl tract but are efficiently hydrolyzed by human gut microbiota in vitro. Molecular Nutrition \& Food Research, 61 (4), 1600680. https:// doi.org/10.1002/mnfr.201600680

Griessler, K., Rodrigues, I., Handl, J. y Hofstetter, U. (2010). Occurrence of mycotoxins in Southern Europe. World Mycotoxin Journal, 3 (3), pp. 301-309. https:// doi.org/10.3920/WMJ2009.1198

Harper, A. F., Estienne, M. J., Meldrum, J. B., Harrell, R. J. y Diaz, D. E. (2010). Assessment of a hydrated sodium calcium aluminosilicate agent and antioxidant blend for mitigation of aflatoxin-induced physiological alterations in pigs. Journal of Swine Health Production, 18 (6), pp. 282-289.

Hope, J. (2013). A review of the mechanism of injury and treatment approaches for illness resulting from exposure to water-damaged buildings, mold, and mycotoxins. The Scientific World Journal, 2013, 767482. https://doi. org/10.1155/2013/767482

Howard, P. C., Churchwell, M. I., Couch, L. H., Marques, M. M. y Doerge, D. R. (1998). Formation of $\mathrm{N}$-(carboxymethyl)-fumonisin B1, following the reaction of fumonisin B1 with reducing sugars. Journal of Agricultural and Food Chemistry, 46 (9), pp. 3546-3557. https:// doi.org/10.1021/jf980194q

Jard, G., Liboz, T., Mathieu, F., Guyonvarc'h, A. y Lebrihi, A. (2011). Review of mycotoxin reduction in food and feed: From prevention in the field to detoxification by adsorption or transformation. Food Additives \& Contaminants: Part A, 28 (11), pp. 1590-1609. https://doi.org/10. 1080/19440049.2011.595377

Kolpin, D. W., Schenzel, J., Meyer, M. T., Phillips, P. J., Hubbard, L. E., Scott, T. M. y Bucheli, T. D. (2014). Mycotoxins: diffuse and point source contributions of natural contaminants of emerging concern to streams. Science of the Total Environment, 470-471, pp. 669676. https://doi.org/10.1016/j.scitotenv.2013.09.062

Kong, C., Shin, S. Y. y Kim, B. G. (2014). Evaluation of mycotoxin sequestering agents for aflatoxin and deoxynivalenol: an in vitro approach. SpringerPlus, 3, 346. https://doi.org/10.1186/21931801-3-346

Koynarski, V., Stoev, S., Grozeva, N., Mirtcheva, T., Daskalov, H., Mitev, J. y Mantle, P. (2007). Experimental coccidiosis provoked by Eimeria acervulina in chicks simultaneously fed on ochratoxin A contaminated diet. Research in Veterinary Science, 82 (2), pp. 225-231. https://doi. org/10.1016/j.rvsc.2006.07.004

Le, T. H., Alassane-Kpembi, I., Oswald, I. P. y Pinton, P. (2017). Analysis of the interactions between environmental and food contaminants, cadmium and deoxynivalenol, in different target organs. Science of the Total Environment, 622-623, pp. 841-848. https://doi.org/10.1016/j.scitotenv.2017.12.014

Lewis L., Onsongo M., Njapau H., SchurzRogers, H., Luber G., Kieszak S., Nyamongo J. [...] y Rubin C. (2005). Aflatoxin contamination of commercial maize products during an outbreak of acute aflatoxicosis in Eastern and Central Kenya. Environmental Health Perspectives, 113 (12), pp. 1763-1767. https://doi. org/10.1289/ehp.7998

Logrieco, A. F. y Moretti, A. (2008). Between emerging and historical problems: An overview of the main toxigenic fungi and mycotoxin concerns in Europe. En: Leslie, J. F., Brandyopadhyay, R. y Visconti, A. (eds.) Mycotoxins: Detection methods, management, public health and agricultural trade. Wallingford: CABI. https://doi. org/10.1079/9781845930820.0139

Madgwick, J. W., West, J. S., White, R. P., Semenov, M. A., Townsend, J. A., Turner, J. A. y Fitt, B. D. L. (2011). Impacts of climate change on wheat anthesis and fusarium ear blight in the UK. European Journal of Plant Pathology, 130 (1), pp. 117-131. https://doi.org/10.1007/ s10658-010-9739-1

Marin, S., Cano-Sancho, G., Sanchis, V. y Ramos, A. J. (2018). The role of mycotoxins in the Human Exposome: application of mycotoxin biomarkers in exposomehealth studies. Food and Chemical Toxicology, 121, pp. 504-518. https://doi. org/10.1016/j.fct.2018.09.039

Marin, S., Ramos, A. J., Cano-Sancho G. y Sanchis V. (2013). Mycotoxins: Occurrence, toxicology, and exposure assessment. Food and Chemical Toxicology, 60, pp. 218-237. https://doi. org/10.1016/j.fct.2013.07.047

Medina, A., González-Jartin, J. M. y Sainz, M. J. (2017). Impact of global warming on mycotoxins. Current Opinion in Food Science, 18, pp. 76-81. https://doi. org/10.1016/j.cofs.2017.11.009

Milani, J. y Maleki, G. (2014). Effects of processing on mycotoxin stability in cereals. Journal of the Science of Food and Agriculture, 94 (12), pp. 2372-2375. https://doi.org/10.1002/jsfa.6600

Nielsen, L. K., Jensen, J. D., Nielsen, G. C., Jensen, J. E., Spliid, N. H., Thomsen, I. K., Justesen, A. F., Collinge, D. B. y Jørgensen, L. N. (2011). Fusarium head blight of cereals in Denmark: Species complex and related mycotoxins. Phytopathology, 101 (8), pp. 960-969. https://doi. org/10.1094/РНYTO-07-10-0188

Pestka, J. J. y Bondy, G. S. (1990). Alteration of immune function following dietary mycotoxin exposure. Canadian Journal of Physiology and Pharmacology, 68 (7), pp. 1009-1016. https://doi. org/10.1139/y90-154

Ramos, A. J. y Hernández, E. (1997). Prevention of aflatoxicosis in farm animals by means of hydrated sodium calcium aluminosilicate addition to feedstuffs: A review. Animal Feed Science and Technology, 65 (1-4), pp. 197-206. https://doi. org/10.1016/S0377-8401(96)01084-X

Ramos, A. J., Da Rocha Rosa, C. A., Cavaglieri, L. R. y Guedes, C. A. (2011). Legislación e impacto económico de las micotoxinas. En: Ramos, A. J. (ed.). Micotoxinas y micotoxicosis. Madrid: AMV Ediciones, pp. 427-462.

Rodríguez-Carrasco, Y., Moltó, J. C., Mañes, J. y Berrada, H. (2014). Exposure assessment approach through mycotoxin/creatinine ratio evaluation in uri- 
ne by GC-MS/MS. Food and Chemical Toxicology, 72, pp. 69-75. https://doi. org/10.1016/j.fct.2014.07.014

Rychlik, M., Humpf, H-U., Marko, D., Dänicke, S., Mally, A., Berthiller, Klaffke, H. y Lorenz, N. (2014). Proposal of a comprehensive definition of modified and other forms of mycotoxins including "masked" mycotoxins. Mycotoxin Research, 30 (4), pp. 197-205. https://doi. org/10.1007/s12550-014-0203-5

Samar, M., Neira, M., Resnik, S. y Pacin, A. (2001). Effect of fermentation on naturally occurring deoxynivalenol (DON) in Argentinean baking process in the bread processing technology. Food Additives \& Contaminants, 18 (11), pp. 1004-1010. https:// doi.org/10.1080/02652030110051284

Smith, M. C., Madec, S., Coton, E. y Hymery, N. (2016). Natural co-occurrence of mycotoxins in foods and feeds and their in vitro combined toxicological effects. Toxins, 8 (4), pp. 94-130. https://doi. org/10.3390/toxins8040094

Solfrizzo, M., Gambacorta, L. y Visconti, A. (2014). Assessment of multi-mycotoxin exposure in southern Italy by urinary multi-biomarker determination. Toxins, 6 (2), pp. 523-538. https://doi. org/10.3390/toxins6020523

Stoev, S. D., Goundasheva, D., Mirtcheva, T. y Mantle, P. G. (2000). Susceptibility to secondary bacterial infections in growing pigs as an early response in ochratoxicosis. Experimental and Toxicologic Pathology, 52 (4), pp. 287-296. https://doi. org/10.1016/S0940-2993(00)80049-4

Traore, T., Bechaux, C., Sirot, V. y Crepet, A. (2016). To which chemical mixtures are the French population exposed? Mixture identification from the second French Total Diet Study. Food Chemistry and Toxicology, 98, pp. 179-188. https://doi. org/10.1016/j.fct.2016.10.028 van der Fels-Klerx, H. J., van Asselt, E. D., Madsen, M. S. y Olesen, J. E. (2013). Impact of climate change effects on contamination of cereal grains with deoxynivalenol. PloS one, 8 (9), e73602. https:// doi.org/10.1371/journal.pone.0073602

Vidal, A., Ambrosio, A., Sanchis, V., Ramos, A. J. y Marín, S. (2016). Enzyme bread improvers affect the stability of deoxynivalenol and deoxynivalenol-3-glucoside during breadmaking. Food Chemistry, 208, pp. 288-296. https://doi. org/10.1016/j.foodchem.2016.04.003

Vidal, A., Morales, H., Sanchis, V., Ramos, A. J. y Marín, S. (2014). Stability of DON and OTA during the breadmaking process and determination of process and performance criteria. Food Control, 40, pp. 234-242. https://doi.org/10.1016/j. foodcont.2013.11.044

Vila-Donat, P., Marín, S., Sanchis, V. y Ramos, A. J. (2018). A review of the mycotoxin adsorbing agents, with an emphasis on their multi-binding capacity, for animal feed decontamination. Food and Chemical Toxicology, 114, pp. 246-259. https://doi.org/10.1016/j. fct.2018.02.044

Wallin, S., Gambacorta, L., Kotova, N., Warensjö Lemming, E., Nälsén, C., Solfrizzo, M. y Olsen, M. (2015). Biomonitoring of concurrent mycotoxin exposure among adults in Sweden through urinary multi-biomarker analysis. Food and Chemical Toxicology, 83, pp. 133-139. https://doi.org/10.1016/j. fct.2015.05.023

Wielogórska, E., MacDonald, S. y Elliot C. T. (2016). A review of the efficacy of mycotoxin detoxifying agents used in feed in light of changing global environment and legislation. World Mycotoxin Journal, 9 (3), pp. 419-433. https://doi. org/10.3920/WMJ2015.1919

\section{Recursos en línea}

Commission regulation (EC) No. 386/2009 of 12 May 2009 amending Regulation (EC) No. 1831/2003 of the European Parliament and of the Council as regards the establishment of a new functional group of feed additives. Official Journal of the European Union, L 118, 66. [En línea]. Disponible en: https://eur-lex. europa.eu/LexUriServ/LexUriServ.do?u ri=OJ:L:2009:118:0066:0066:EN:PDF

Manual on the application of the HACCP system in mycotoxin prevention and control. FAO. Food and Nutrition Paper 73, 2001. [En línea]. Disponible en: http://www.fao.org/3/a-y1390e.pdf

Opinion of the Scientific Panel on Contaminants in the Food Chain on a request from the Commission related to the potential increase of consumer health risk by a possible increase of the existing maximum levels for aflatoxins in almonds, hazelnuts and pistachios and derived products. EFSA Journal, 5 (3), 446, 2007. [En línea]. Disponible en: https://efsa.onlinelibrary.wiley.com/ doi/10.2903/j.efsa.2007.446

RASFF. The Rapid Alert System for Food and Feed (2018). RASFF annual report 2017. Luxembourg: Publications Office of the European Union, 2018. [En línea]. https://doi.org/10.2875/767865

The costs of mycotoxins in animal production. All about feed, 23 mayo 2016. [En línea]. Disponible en: https://www. allaboutfeed.net/Mycotoxins/Articles/2016/5/The-cost-of-mycotoxins-inanimal-production-2787006W/ 